\title{
HISTOLOGICAL SUBTYPES OF FOCAL CORTICAL DYSPLASIA
}

Clinical, EEG, MRI, neuropsychological, surgical, and seizure outcome data in 200 children with histologically proven cortical malformations were analyzed in a study at Motol University Hospital, Prague, Czech Republic, Miami Children's Hospital, and other centers. Pathological subtypes included mild malformation of cortical development type II (mMCD) in 36, focal cortical dysplasia (FCD) type Ia in 55 , FCD type Ib in 39, FCD type IIa in 35, and FCD type IIb in 35 subjects. Malformations classified as mMCD I and II have ectopic neurons in layer I or in white matter; FCD la has microcolumnar neurons with ectopic white matter neurons; FCD Ib has giant neurons with cortical dyslamination; FCD IIa has dysmorphic neurons; and FCD IIb has additional balloon cells. Clinical history includes prenatal and perinatal risk factors (eg. prematurity, asphyxia, bleeding and hydrocephalus) in $13 \%$ of the patient cohort. Intrauterine factors in the late second or early third trimester may account for severe FCD (type II), whereas events occurring closer to birth explain milder forms of cortical malformation ( $\mathrm{mMCD}, \mathrm{FCD}$ type I). All malformation subtypes possess a comparable degree of epileptogenicity. Even mildly dysplastic features (eg. mMCD) are highly epileptogenic. EEG abnormalities (fast frequencies and continuous epileptiform discharges) were not specific to individual histopathological subtypes, but FCD IIb had more localized ictal EEG patterns and focal MRI abnormalities, especially FCD type IIb. Lobar hypoplasia/atrophy was common and hippocampal sclerosis was most frequent in FCD type I cases. EEG status occurred in only $9 \%$ of patients. No statistical differences in cognitive functioning were detected in histological subtypes in this study. The extent of surgery was similar in all subtypes. Patients with FCD IIb malformation had the best surgical outcome ( $75 \%$ patients seizure-free), whereas FCD type Ib patients had the poorest outcome ( $45 \%$ seizure-free). Children with $\mathrm{mMCD}$ showed intermediate surgical success with $52 \%$ seizurefree. (Krsek P, Maton B, Korman B, et al. Different features of histopathological subtypes of pediatric focal cortical dysplasia. Ann Neurol June 2008;63:758-769). (Respond: Dr Krsek, Department of Pediatric Neurology, Charles University, Second Medical School, Motol University Hospital, V Uvalu 84, CZ 15006 Prague 5, Czech Republic. E-mail: pavel.krsck(apost.c $)$.

COMMENT. This study confirmed a practical reason for histopathological classification of mMCD, FCD type I, and FCD type II subtypes, but distinctions between ' $a$ ' and 'b' subtypes were minor. EEG and MRI definition of FCD subtypes might influence surgical planning and outcome. Fauser S and associates in Freiburg, Germany, reported a better postsurgical outcome with FCD type I patients (Brain 2004;127:2406-2418). Tassi L et al (Brain 2002;125:1719-1732) used a simpler 3-category classification of FCD, and found that patients with giant dysmorphic neurons and balloon cells (equivalent to subtypes IIa and IIb) had the best outcome, a result similar to that of Krsek et al in Czech Republic.

Environmental causes of CNS maldevelopment, in addition to pre- and perinatal asphyxia and bleeding mentioned above, include toxins, antiepileptic drugs, infections, and genetic factors (Rodier PM. Pediatrics 2004;113:1076-1083; Sarnat HB, Flores-Sarnat L. Eur J Paediatr Neurol 2001;5:57-64; Ped Neur Briefs April 2004;18:29). 